\title{
On special values at integers of $L$-functions of Jacobi theta products of weight 3
}

\author{
Ryojun Ito*
}

\begin{abstract}
In this paper, we consider $L$-functions of modular forms of weight 3 , which are products of the Jacobi theta series, and express their special values at $s=3,4$ in terms of special values of Kampé de Fériet hypergeometric functions. Moreover, via $L$-values, we give some relations between special values of Kampé de Fériet hypergeometric functions and generalized hypergeometric functions.
\end{abstract}

\section{Introduction and Main Results}

Let $f$ be a modular form of weight $k$ with $q$-expansion $f(q)=\sum_{n=0}^{\infty} a_{n} q^{n}$ $\left(q=e^{2 \pi i \tau}, \operatorname{Im}(\tau)>0\right)$. Then its $L$-function $L(f, s)=\sum_{n=1}^{\infty} a_{n} / n^{s}$ converges absolutely on $\operatorname{Re}(s)>k+1(\operatorname{Re}(s)>k / 2+1$ if $f$ is a cusp form). When the Fricke involution image $f^{\#}$ of $f$ is also a modular form, then $L(f, s)$ is meromorphically continued to the whole complex plane with a possible simple pole at $s=k$, and is entire when $f^{\#}(0)=0$ (see [15, Theorem 3.2]). In this paper, we consider the case when $f(q)$ is a product of the Jacobi theta series

$$
\theta_{2}(q):=\sum_{n \in \mathbb{Z}} q^{\left(n+\frac{1}{2}\right)^{2}}, \quad \theta_{3}(q):=\sum_{n \in \mathbb{Z}} q^{n^{2}}, \quad \theta_{4}(q):=\sum_{n \in \mathbb{Z}}(-1)^{n} q^{n^{2}}
$$

which are modular forms of weight $1 / 2$, and satisfies the condition $f^{\#}(0)=0$.

In [13], by an analytic method, Rogers and Zudilin expressed $L(f, 2)$ for some theta products $f(q)$ of weight 2 in terms of special values of generalized hypergeometric functions

$$
{ }_{p+1} F_{p}\left[\begin{array}{c}
a_{1}, a_{2}, \ldots, a_{p+1} \\
b_{1}, b_{2}, \ldots, b_{p}
\end{array} \mid z\right]:=\sum_{n=0}^{\infty} \frac{\left(a_{1}\right)_{n} \cdots\left(a_{p+1}\right)_{n}}{\left(b_{1}\right)_{n} \cdots\left(b_{p}\right)_{n}} \frac{z^{n}}{(1)_{n}}
$$

where $(a)_{n}:=\Gamma(a+n) / \Gamma(a)$ denotes the Pochhammer symbol. Other known results of hypergeometric expressions of $L$-values are the following.

1. Otsubo [10] expressed $L(f, 2)$ for some theta products $f(q)$ of weight 2 in terms of ${ }_{3} F_{2}(1)$ via regulators.

* Department of Mathematics and Informatics, Graduate School of Science, Chiba University, Yayoicho 1-33, Inage, Chiba, 263-8522 Japan. E-mail: afua9032@chiba-u.jp, 2010 Mathematics Subject Classification: 11F27, 11F67, 33C20, 33C99 keywords: theta series, $L$-values for theta products, generalized hypergeometric functions, Kampé de Fériet hypergeometric functions. 
2. Rogers [11], Rogers-Zudilin [13], Zudilin [19] and the author [8] expressed $L(f, 2)$ for some theta products $f(q)$ of weight 2 in terms of ${ }_{3} F_{2}(1)$ by using the Rogers-Zudilin method. Furthermore, Zudilin [19] expressed $L(f, 3)$ for the theta product which corresponds to the elliptic curve of conductor 32 in terms of ${ }_{4} F_{3}(1)$.

3. Rogers-Wan-Zucker [12] expressed $L(f, 2)$ (resp. $L(f, 3), L(f, 4))$ for some quotients $f(q)$ of the Dedekind eta function $\eta(q):=q^{1 / 24} \prod_{n=1}^{\infty}\left(1-q^{n}\right)$ of weight 3 (resp. 4,5 ) in terms of special values of generalized hypergeometric functions or the gamma function by an analytic method. The author [9] expressed $L(f, 1)$ (hence the values at 2 by the functional equations) for some theta products $f(q)$ of weight 3 in terms of ${ }_{3} F_{2}(1)$ by using the Rogers-Zudilin method.

4. Samart [14] expressed $L(f, 3)$ for some eta quotients $f(q)$ of weight 3 in terms of ${ }_{5} F_{4}(1)$ via Mahler measures.

In this paper, we consider the following normalized Jacobi theta products of weight 3

$$
f(q)=\frac{1}{16} \theta_{2}^{4}(q) \theta_{4}^{2}(q), \quad g(q)=\frac{1}{16} \theta_{2}^{4}(q) \theta_{4}^{2}\left(q^{2}\right) .
$$

We remark that $f(q)$ is an Eisenstein series twisted by some Dirichlet characters and $g(q)$ is the cusp form corresponding to the Kummer K3 surface defined by $z^{2}=x\left(x^{2}-1\right) y\left(y^{2}-1\right)$ (cf. [18, Theorem 7.4]).

The aim of this paper is to express $L(f, n)$ and $L(g, n)$ for $n=3,4$ in terms of special values of the Kampé de Fériet hypergeometric function [1, 17]

$$
\begin{aligned}
& F_{C ; D ; D^{\prime}}^{A ; B ; B^{\prime}}\left[\begin{array}{l}
a_{1}, \ldots, a_{A} ; b_{1}, \ldots, b_{B}, b_{1}^{\prime}, \ldots, b_{B^{\prime}}^{\prime} \\
c_{1}, \ldots, c_{C} ; d_{1}, \ldots, d_{D} ; d_{1}^{\prime}, \ldots, d_{D^{\prime}}^{\prime}
\end{array} \mid x, y\right] \\
& :=\sum_{m, n=0}^{\infty} \frac{\prod_{i=1}^{A}\left(a_{i}\right)_{m+n} \prod_{i=1}^{B}\left(b_{i}\right)_{m} \prod_{i=1}^{B^{\prime}}\left(b_{i}^{\prime}\right)_{n}}{\prod_{i=1}^{C}\left(c_{i}\right)_{m+n} \prod_{i=1}^{D}\left(d_{i}\right)_{m} \prod_{i=1}^{D^{\prime}}\left(d_{i}^{\prime}\right)_{n}} \frac{x^{m} y^{n}}{(1)_{m}(1)_{n}},
\end{aligned}
$$

which is a two-variable generalization of generalized hypergeometric functions.

The main results are the following.

Theorem 1. 1.

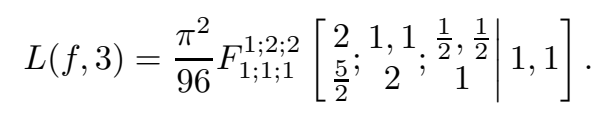

2.

$$
L(g, 3)=\frac{\pi^{3}}{128} F_{1 ; 1 ; 1}^{1 ; 2 ; 2}\left[\begin{array}{c}
\frac{3}{2} \\
2
\end{array} \frac{2}{2}, \frac{3}{2} ; \frac{1}{2}, \frac{1}{2} \mid 1,1\right]
$$

Theorem 2. 1.

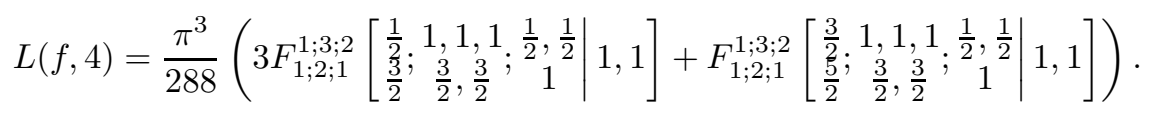

2.

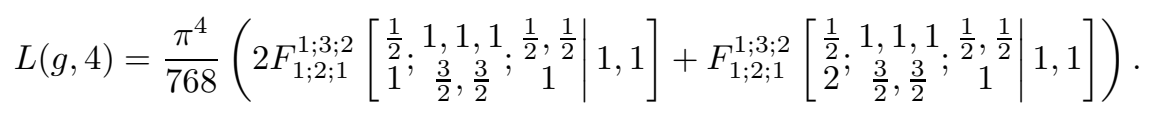


We remark that the double series $F_{A: B: C}^{A: B: C+1}(x, y)$ converges absolutely on $|x| \leqq 1$ and $|y| \leqq 1$ when the parameters satisfy the following conditions [7, Theorem 1]

$$
\begin{aligned}
& \operatorname{Re}\left(\sum_{i=1}^{A} c_{i}+\sum_{i=1}^{B} d_{i}-\sum_{i=1}^{A} a_{i}-\sum_{i=1}^{B+1} b_{i}\right)>0, \\
& \operatorname{Re}\left(\sum_{i=1}^{A} c_{i}+\sum_{i=1}^{C} d_{i}^{\prime}-\sum_{i=1}^{A} a_{i}-\sum_{i=1}^{C+1} b_{i}^{\prime}\right)>0, \\
& \operatorname{Re}\left(\sum_{i=1}^{A} c_{i}+\sum_{i=1}^{B} d_{i}+\sum_{i=1}^{C} d_{i}^{\prime}-\sum_{i=1}^{A} a_{i}-\sum_{i=1}^{B+1} b_{i}-\sum_{i=1}^{C+1} b_{i}^{\prime}\right)>0 .
\end{aligned}
$$

We prove Theorems 1 and 2 by using the Rogers-Zudilin method. The strategy of the method is as follows. For $h(q)=\sum_{n=0}^{\infty} a_{n} q^{n}$ and $n \in \mathbb{Z}_{\geqq 1}$, the value $L(h, n)$ is obtained by the Mellin transformation of $h(q)$

$$
L(h, n)=\frac{(-1)^{n-1}}{\Gamma(n)} \int_{0}^{1}\left(h(q)-a_{0}\right)(\log q)^{n-1} \frac{d q}{q} .
$$

The key to express the value $L(h, n)$ in terms of special values of hypergeometric functions is the following transformation formulas

$$
\theta_{3}^{2}(q)={ }_{2} F_{1}\left[\begin{array}{c}
\frac{1}{2}, \frac{1}{2} \\
1
\end{array} \mid \alpha\right], \quad \theta_{3}^{4}(q) \frac{d q}{q}=\frac{d \alpha}{\alpha(1-\alpha)},
$$

where $\alpha:=\theta_{2}^{4}(q) / \theta_{3}^{4}(q)$. The former is [3, p.101, Entry 6], and the latter follows from the former and [2, p.87, Entry 30]. By these formulas, we reduce the integral (1) to an integral of the form

$$
\int_{0}^{1} P(\alpha)_{p+1} F_{p}\left[\begin{array}{c}
a_{1}, a_{2}, \ldots, a_{p+1} \\
b_{1}, \ldots, b_{p}
\end{array} \mid \alpha\right]{ }_{2} F_{1}\left[\begin{array}{c}
\frac{1}{2}, \frac{1}{2} \\
1
\end{array} \mid \alpha\right] \frac{d \alpha}{\alpha(1-\alpha)}
$$

where $P(\alpha)$ is a polynomial in $\alpha^{k}(1-\alpha)^{l}$ for various $k$ and $l$. Then, by simple computations, we obtain hypergeometric expressions of $L$-values.

Finally, we remark that we have simpler hypergeometric expressions of the $L$-values $L(f, 3), L(f, 4)$ and $L(g, 3)$. Since $f(q)$ is the Eisenstein series twisted by the Dirichlet characters $\chi_{-4}(n):=\operatorname{Im}\left(i^{n}\right)$ and $\psi(n):=(-1)^{n-1}[5$, p. 281, Lemma $3.32(3.85)$ ]

$$
f(q)=\frac{1}{16} \theta_{2}^{4}(q) \theta_{4}^{2}(q)=\sum_{n=1}^{\infty} \frac{(-1)^{n+1} n^{2} q^{n}}{1+q^{2 n}}=\sum_{n, k=1}^{\infty} \psi(n) n^{2} \chi_{-4}(k) q^{n k}
$$

we have

$$
L(f, s)=\sum_{n, k=1}^{\infty} \frac{\psi(n) n^{2} \chi_{-4}(k)}{n^{s} k^{s}}=L(\psi, s-2) L\left(\chi_{-4}, s\right),
$$

where $L(\chi, s)$ is the Dirichlet $L$-function associated to a Dirichlet character $\chi$. We know $L(\psi, 1)=\log 2$ and $L\left(\chi_{-4}, 3\right)=\pi^{3} / 32$, hence we obtain

$$
L(f, 3)=\frac{\pi^{3} \log 2}{32} .
$$


Moreover, the value $L(f, 4)$ can be expressed in terms of ${ }_{5} F_{4}$, since we have $L(\psi, 2)=\pi^{2} / 12$ and

$L\left(\chi_{-4}, 4\right)={ }_{5} F_{4}\left[\begin{array}{c}\frac{1}{2}, \ldots, \frac{1}{2}, 1 \\ \frac{3}{2}, \ldots, \frac{3}{2}\end{array} \mid-1\right]={ }_{5} F_{4}\left[\begin{array}{c}\frac{1}{4}, \ldots, \frac{1}{4}, 1 \\ \frac{5}{4}, \ldots, \frac{5}{4}\end{array} \mid 1\right]-\frac{1}{81}{ }_{5} F_{4}\left[\begin{array}{c}\frac{3}{4}, \ldots, \frac{3}{4}, 1 \\ \frac{7}{4}, \ldots, \frac{7}{4}\end{array} \mid 1\right]$,

which easily follows from

$$
2 n+1=\frac{\left(\frac{3}{2}\right)_{n}}{\left(\frac{1}{2}\right)_{n}}, \quad 4 n+1=\frac{\left(\frac{5}{4}\right)_{n}}{\left(\frac{1}{4}\right)_{n}}, \quad 4 n+3=\frac{3\left(\frac{7}{4}\right)_{n}}{\left(\frac{3}{4}\right)_{n}} .
$$

Similarly, we have a simpler expression of $L(g, 3)$ [14, Corollary 1.3]

$$
L(g, 3)=\frac{\pi^{3}}{1024}\left(48 \log 2-{ }_{5} F_{4}\left[\begin{array}{c|c}
\frac{3}{2}, \frac{3}{2}, \frac{3}{2}, 1,1 & 1 \\
2,2,, 2,2 & 1
\end{array}\right]\right) .
$$

Therefore we obtain via $L$-values the following reduction formulas for Kampé de Fériet hypergeometric functions.

Corollary 3. 1.

$$
F_{1 ; 1 ; 1}^{1 ; 2 ; 2}\left[\begin{array}{l}
\frac{2}{2} \\
\frac{5}{2}
\end{array} \underset{2}{1,1} ; \frac{1}{2}, \frac{1}{2} \mid 1,1\right]=3 \pi \log 2
$$

2.

$$
8 F_{1 ; 1 ; 1}^{1 ; 2 ; 2}\left[\frac{\frac{3}{2}}{2} ;{ }_{\frac{3}{2}}^{\frac{1}{2}}, 1+\frac{1}{2}, \frac{1}{2} \mid 1,1\right]=48 \log 2-{ }_{5} F_{4}\left[\begin{array}{c}
\frac{3}{2}, \frac{3}{2}, \frac{3}{2}, 1,1 \\
2,2,2,2,2
\end{array}\right]
$$

3.

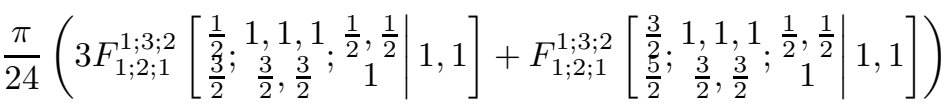

$$
\begin{aligned}
& ={ }_{5} F_{4}\left[\begin{array}{c}
\frac{1}{2}, \ldots, \frac{1}{2}, 1 \\
\frac{3}{2}, \ldots, \frac{3}{2}
\end{array} \mid-1\right] \text {. }
\end{aligned}
$$

The author does not know how to derive these formulas directly. It is new that the value $L(g, 4)$ is expressed in terms of special values of hypergeometric functions. Similarly to the results above, one might be able to express the value $L(g, 4)$ in terms of special values of generalized hypergeometric functions.

\section{Proof of Theorem 1}

We first show the following integral expressions of the $L$-values $L(f, 3)$ and $L(g, 3)$.

Proposition 4. 1.

$$
L(f, 3)=\frac{\pi^{2}}{8} \int_{0}^{1} \theta_{2}^{4}(q) \theta_{4}^{2}(q) \sum_{r, k=1}^{\infty} \frac{q^{(2 r-1)(2 k-1)}}{2 r-1} \frac{d q}{q} .
$$

2.

$$
L(g, 3)=\frac{\pi^{2}}{16} \int_{0}^{1} \theta_{2}^{4}(q) \theta_{4}^{2}(q) \sum_{r, k=1}^{\infty} \frac{q^{2(r-1 / 2)(k-1 / 2)}}{2 r-1} \frac{d q}{q}
$$


Proof. We prove the formula for $L(f, 3)$ only. Similar computation leads to the remaining formula.

By (1), we have

$$
L(f, 3)=\frac{1}{2} \int_{0}^{1} \frac{1}{16} \theta_{2}^{4}(q) \theta_{4}^{2}(q)(\log q)^{2} \frac{d q}{q} .
$$

By changing the variable $q=e^{-\pi u}$, we have

$$
L(f, 3)=\frac{\pi^{3}}{32} \int_{0}^{\infty} \theta_{2}^{4}\left(e^{-\pi u}\right) \theta_{4}^{2}\left(e^{-\pi u}\right) u^{2} d u .
$$

If we use the involution formula for $\theta_{4}(q)[4, \mathrm{p} .40,(2.3 .3)]$, the Lambert series expansions of $\theta_{2}^{2}(q)$ and $\theta_{2}^{4}(q)[5$, p.177, Theorem $3.10(3.15)$ and p.196, Theorem $3.26(3.69)]$

$$
\begin{aligned}
\sqrt{u} \theta_{4}\left(e^{-\pi u}\right) & =\theta_{2}\left(e^{-\frac{\pi}{u}}\right), \\
\theta_{2}^{2}(q) & =4 \sum_{n, k=1}^{\infty} \chi_{-4}(n) q^{n(k-1 / 2)}, \quad \chi_{-4}(n):=\operatorname{Im}\left(i^{n}\right), \\
\theta_{2}^{4}(q) & =16 \sum_{r, s=1}^{\infty}(2 r-1) q^{(2 r-1)(2 s-1)}
\end{aligned}
$$

and the substitution $u \mapsto n u /(2 r-1)$, then we obtain

$$
L(f, 3)=2 \pi^{3} \int_{0}^{\infty}\left(\sum_{n, s=1}^{\infty} \chi_{-4}(n) n^{2} e^{-\pi u n(2 s-1)}\right)\left(\sum_{r, k=1}^{\infty} \frac{e^{-\frac{\pi(2 r-1)(k-1 / 2)}{u}}}{2 r-1}\right) u d u .
$$

We know that the first series in the integral above is the theta product [5, Lemma $3.32(3.84)$ ]

$$
\sum_{n, s=1}^{\infty} \chi_{-4}(n) n^{2} q^{n(2 s-1)}=\frac{1}{4} \theta_{2}^{2}\left(q^{2}\right) \theta_{4}^{4}\left(q^{2}\right)
$$

By this identity and (3), we have

$$
\begin{aligned}
L(f, 3) & =\frac{\pi^{3}}{2} \int_{0}^{\infty} \theta_{2}^{2}\left(e^{-2 \pi u}\right) \theta_{4}^{4}\left(e^{-2 \pi u}\right)\left(\sum_{r, k=1}^{\infty} \frac{e^{-\frac{\pi(2 r-1)(k-1 / 2)}{u}}}{2 r-1}\right) u d u \\
& =\frac{\pi^{3}}{16} \int_{0}^{\infty} \theta_{4}^{2}\left(e^{-\pi / 2 u}\right) \theta_{2}^{4}\left(e^{-\pi / 2 u}\right)\left(\sum_{r, k=1}^{\infty} \frac{e^{-\frac{\pi(2 r-1)(k-1 / 2)}{u}}}{2 r-1}\right) \frac{d u}{u^{2}} .
\end{aligned}
$$

If we use the substitutions $u \mapsto 1 / u, q=e^{-\pi u}$ and $q \mapsto q^{2}$, then we obtain the formula.

The series in the integrals in Proposition 4 are hypergeometric functions.

Lemma 5. 1.

$$
\sum_{r, k=1}^{\infty} \frac{q^{(2 r-1)(2 k-1)}}{2 r-1}=\frac{\alpha}{16}{ }_{2} F_{1}\left[\begin{array}{c}
1,1 \\
2
\end{array} \mid \alpha\right]
$$


2.

$$
\sum_{r, k=1}^{\infty} \frac{q^{2(r-1 / 2)(k-1 / 2)}}{2 r-1}=\frac{\alpha^{1 / 2}}{4}{ }_{2} F_{1}\left[\begin{array}{c}
\frac{1}{2}, 1 \\
\frac{3}{2}
\end{array} \mid \alpha\right] .
$$

Proof. We prove these hypergeometric expressions by using the transformation formulas (2).

By (5), we have

$$
\sum_{r, k=1}^{\infty} \frac{q^{(2 r-1)(2 k-1)}}{2 r-1}=\int_{0}^{q} \sum_{r, k=1}^{\infty}(2 k-1) q^{(2 r-1)(2 k-1)} \frac{d q}{q}=\frac{1}{16} \int_{0}^{q} \theta_{2}^{4}(q) \frac{d q}{q} .
$$

If we use (2), then the integral above is equal to

$$
\frac{1}{16} \int_{0}^{\alpha} \alpha \frac{d \alpha}{\alpha(1-\alpha)}=-\frac{1}{16} \log (1-\alpha) .
$$

Since we know

$$
-\log (1-\alpha)=\alpha_{2} F_{1}\left[\begin{array}{c|c}
1,1 \\
2
\end{array} \mid \alpha\right]
$$

we obtain the first formula.

Similarly, we have

$$
\sum_{r, k=1}^{\infty} \frac{q^{2(r-1 / 2)(k-1 / 2)}}{2 r-1}=\frac{1}{32} \int_{0}^{q} \theta_{2}^{4}\left(q^{\frac{1}{2}}\right) \frac{d q}{q}=\frac{1}{8} \int_{0}^{q} \theta_{2}^{2}(q) \theta_{3}^{2}(q) \frac{d q}{q} .
$$

Here we used $2 \theta_{2}\left(q^{2}\right) \theta_{3}\left(q^{2}\right)=\theta_{2}^{2}(q)[3$, p. 40 , Entry 25 (iv)] for the last equality. Then, by (2), we obtain

$$
\int_{0}^{q} \theta_{2}^{2}(q) \theta_{3}^{2}(q) \frac{d q}{q}=\int_{0}^{\alpha} \alpha^{\frac{1}{2}} \frac{d \alpha}{\alpha(1-\alpha)} .
$$

By the integral representation of hypergeometric functions $[16,(1.6 .6)]$

$$
{ }_{2} F_{1}\left[\begin{array}{c}
a, b \\
c
\end{array} \mid z\right]=\frac{\Gamma(c)}{\Gamma(b) \Gamma(c-b)} \int_{0}^{1} t^{b}(1-t)^{c-b}(1-z t)^{-a} \frac{d t}{t(1-t)},
$$

we have

$$
\begin{aligned}
\int_{0}^{\alpha} t^{\frac{1}{2}} \frac{d t}{t(1-t)} & =\alpha^{\frac{1}{2}} \int_{0}^{1} t^{\frac{1}{2}}(1-t)(1-\alpha t)^{-1} \frac{d t}{t(1-t)} \\
& =\alpha^{\frac{1}{2}} \frac{\Gamma\left(\frac{1}{2}\right) \Gamma(1)}{\Gamma\left(\frac{3}{2}\right)}{ }_{2} F_{1}\left[\begin{array}{c}
\frac{1}{2}, 1 \\
\frac{3}{2}
\end{array} \mid \alpha\right],
\end{aligned}
$$

hence we obtain the second formula.

Proof of Theorem 1. By Lemma 5 and the transformation formulas (2), we have

$$
\begin{aligned}
& L(f, 3)=\frac{\pi^{2}}{128} \int_{0}^{1} \alpha^{2}(1-\alpha)^{1 / 2}{ }_{2} F_{1}\left[\begin{array}{c}
1,1 \\
2
\end{array} \mid \alpha\right]{ }_{2} F_{1}\left[\begin{array}{c}
\left.\frac{1}{2}, \frac{1}{2} \mid \alpha\right] \frac{d \alpha}{1} \mid \alpha(1-\alpha) \\
1
\end{array}\right. \\
& L(g, 3)=\frac{\pi^{2}}{64} \int_{0}^{1} \alpha^{3 / 2}(1-\alpha)^{1 / 2}{ }_{2} F_{1}\left[\begin{array}{c}
\frac{1}{2}, 1 \\
\frac{3}{2}
\end{array} \mid \alpha\right]{ }_{2} F_{1}\left[\begin{array}{c}
\frac{1}{2}, \frac{1}{2} \mid \\
1
\end{array} \mid \alpha\right] \frac{d \alpha}{\alpha(1-\alpha)} .
\end{aligned}
$$


If we use the series expansions of hypergeometric functions and integrate termby-term, we obtain

$$
\begin{aligned}
& L(f, 3)=\frac{\pi^{2}}{128} \int_{0}^{1} \alpha^{2}(1-\alpha)^{1 / 2}{ }_{2} F_{1}\left[\begin{array}{c}
1,1 \\
2
\end{array} \mid \alpha\right]{ }_{2} F_{1}\left[\begin{array}{c}
\frac{1}{2}, \frac{1}{2} \\
1
\end{array} \mid \alpha\right] \frac{d \alpha}{\alpha(1-\alpha)} \\
& =\frac{\pi^{2}}{128} \sum_{m, n=0}^{\infty} \frac{(1)_{m}^{2}}{(2)_{m}(1)_{m}} \frac{\left(\frac{1}{2}\right)_{n}^{2}}{(1)_{n}^{2}} \int_{0}^{1} \alpha^{2+m+n}(1-\alpha)^{\frac{1}{2}} \frac{d \alpha}{\alpha(1-\alpha)} \\
& =\frac{\pi^{2}}{128} \sum_{m, n=0}^{\infty} \frac{(1)_{m}^{2}}{(2)_{m}(1)_{m}} \frac{\left(\frac{1}{2}\right)_{n}^{2}}{(1)_{n}^{2}} \frac{\Gamma(2+m+n) \Gamma\left(\frac{1}{2}\right)}{\Gamma\left(\frac{5}{2}+m+n\right)} \\
& =\frac{\pi^{2}}{96} F_{1 ; 1 ; 1}^{1 ; 2 ; 2}\left[\begin{array}{l}
2 \\
\frac{5}{2}
\end{array} ; 2,1, \frac{1}{2}, \frac{1}{2} \mid 1,1\right] .
\end{aligned}
$$

By similar computations, we have the hypergeometric expression of the value $L(g, 3)$.

\section{Proof of Theorem 2}

Similarly to the computations in the proof of Proposition 4, we obtain the following integral expressions of the $L$-values $L(f, 4)$ and $L(g, 4)$.

Proposition 6. 1.

$$
L(f, 4)=\frac{\pi^{3}}{48} \int_{0}^{1}\left(2 \theta_{4}^{8}\left(q^{2}\right)-\theta_{4}^{8}(q)\right)\left(\sum_{n, r=1}^{\infty} \frac{\chi_{-4}(n)}{(2 r-1)^{2}} q^{n(r-1 / 2)}\right) \frac{d q}{q} .
$$

2.

$$
L(g, 4)=\frac{\pi^{3}}{48} \int_{0}^{1}\left(2 \theta_{4}^{8}\left(q^{4}\right)-\theta_{4}^{8}\left(q^{2}\right)\right)\left(\sum_{n, r=1}^{\infty} \frac{\chi_{-4}(n)}{(2 r-1)^{2}} q^{n(r-1 / 2)}\right) \frac{d q}{q} .
$$

Proof. We prove the formula for $L(f, 4)$ only. The formula for $L(g, 4)$ is obtained by similar computations.

By (1), we have

$$
L(f, 4)=\frac{1}{6} \int_{0}^{1} \frac{1}{16} \theta_{2}^{4}(q) \theta_{4}^{2}(q)(\log q)^{3} \frac{d q}{q}=\frac{\pi^{4}}{96} \int_{0}^{\infty} \theta_{2}^{4}\left(e^{-\pi u}\right) \theta_{4}^{2}\left(e^{-\pi u}\right) u^{3} d u .
$$

By (3), (4), (5) and the variable transformation $u \mapsto(k-1 / 2) u /(2 r-1)$, we have

$L(f, 4)=\frac{\pi^{4}}{12} \int_{0}^{\infty}\left(\sum_{s, k=1}^{\infty}(2 k-1)^{3} e^{\frac{-\pi u(2 s-1)(2 k-1)}{2}}\right)\left(\sum_{n, r=1}^{\infty} \frac{\chi_{-4}(n)}{(2 r-1)^{2}} e^{\frac{-\pi n(2 r-1)}{u}}\right) u^{2} d u$.

The first series in the integral is a theta product.

\section{Lemma 7.}

$$
\sum_{s, k=1}^{\infty}(2 k-1)^{3} q^{(2 s-1)(2 k-1)}=\frac{\theta_{2}^{8}\left(q^{1 / 2}\right)-8 \theta_{2}^{8}(q)}{256}
$$


Proof. We have

$$
\begin{aligned}
\sum_{s, k=1}^{\infty}(2 k-1)^{3} q^{(2 s-1)(2 k-1)} & =\sum_{s, k=1}^{\infty}\left(k^{3} q^{s k}-9 k^{3} q^{2 s k}+8 k^{3} q^{4 s k}\right) \\
& =\frac{M(q)-9 M\left(q^{2}\right)+8 M\left(q^{4}\right)}{240}
\end{aligned}
$$

where

$$
M(q):=1+240 \sum_{s, k=1}^{\infty} k^{3} q^{s k} .
$$

We know that $\theta_{2}(q)$ has the connection with $M(q)$ [5, p.207, Theorem 3.39 (3.101)]

$$
\theta_{2}^{8}\left(q^{\frac{1}{2}}\right)=\frac{16}{15}\left(M(q)-M\left(q^{2}\right)\right)
$$

By this identity, we obtain the lemma.

If we use the lemma above and (3), we have

$$
\begin{aligned}
L(f, 4) & =\frac{\pi^{4}}{3072} \int_{0}^{\infty}\left(\theta_{2}^{8}\left(e^{-\pi u / 4}\right)-8 \theta_{2}^{8}\left(e^{-\pi u / 2}\right)\right)\left(\sum_{n, r=1}^{\infty} \frac{\chi_{-4}(n)}{(2 r-1)^{2}} e^{\frac{-\pi n(2 r-1)}{u}}\right) u^{2} d u \\
& =\frac{\pi^{4}}{24} \int_{0}^{\infty}\left(2 \theta_{4}^{8}\left(e^{-4 \pi / u}\right)-\theta_{4}^{8}\left(e^{-2 \pi / u}\right)\right)\left(\sum_{n, r=1}^{\infty} \frac{\chi_{-4}(n)}{(2 r-1)^{2}} e^{\frac{-\pi n(2 r-1)}{u}}\right) \frac{d u}{u^{2}}
\end{aligned}
$$

By changing the variables $u \mapsto 1 / u$ and $q=e^{-2 \pi u}$, we obtain the proposition.

We remark that the series in the integrals in Proposition 6 can be expressed in terms of generalized hypergeometric functions [6, (2.2)]

$$
\sum_{n, r=1}^{\infty} \frac{\chi_{-4}(n)}{(2 r-1)^{2}} q^{n(r-1 / 2)}=\frac{\alpha^{1 / 2}{ }_{3} F_{2}\left[\begin{array}{c}
1,1,1 \\
\frac{3}{2}, \frac{3}{2}
\end{array} \mid \alpha\right]}{4} .
$$

Proof of Theorem 2. By (2) and (6), we obtain

$$
\begin{aligned}
& L(f, 4)=\frac{\pi^{3}}{192} \int_{0}^{1}\left(\alpha^{1 / 2}+\alpha^{3 / 2}\right)(1-\alpha)_{3} F_{2}\left[\begin{array}{c}
1,1,1 \\
\frac{3}{2}, \frac{3}{2}
\end{array} \mid \alpha\right]{ }_{2} F_{1}\left[\begin{array}{c}
\frac{1}{2}, \frac{1}{2} \\
1
\end{array} \mid \alpha\right] \frac{d \alpha}{\alpha(1-\alpha)} \\
& L(g, 4)=\frac{\pi^{3}}{384} \int_{0}^{1}\left((1-\alpha)^{1 / 2}+(1-\alpha)^{3 / 2}\right) \alpha^{1 / 2}{ }_{3} F_{2}\left[\begin{array}{c}
1,1,1 \\
\frac{3}{2}, \frac{3}{2}
\end{array} \mid \alpha\right]{ }_{2} F_{1}\left[\begin{array}{c}
\frac{1}{2}, \frac{1}{2} \\
1
\end{array} \mid \alpha\right] \frac{d \alpha}{\alpha(1-\alpha)} .
\end{aligned}
$$

Here we used

$$
\begin{aligned}
2 \theta_{4}^{8}\left(q^{2}\right)-\theta_{4}^{8}(q) & =(1+\alpha)(1-\alpha) \theta_{3}^{8}(q), \\
2 \theta_{4}^{8}\left(q^{4}\right)-\theta_{4}^{8}\left(q^{2}\right) & =\frac{1}{2}\left((1-\alpha)^{1 / 2}+(1-\alpha)^{3 / 2}\right) \theta_{3}^{8}(q),
\end{aligned}
$$

which follow from the formulas [4, p. 34, (2.1.7i), (2.1.7ii)]

$$
2 \theta_{3}^{2}\left(q^{2}\right)=\theta_{3}^{2}(q)+\theta_{4}^{2}(q), \quad \theta_{3}(q) \theta_{4}(q)=\theta_{4}^{2}\left(q^{2}\right) .
$$

Then the hypergeometric expressions can be proved by interchanging of the order of summation and integration. 


\section{Acknowledgment}

The author expresses his gratitude to Noriyuki Otsubo for a lot of helpful comments on a draft version of this paper.

\section{References}

[1] P. Appell, J. Kampé de Fériet, Fonctions hypergéométriques et hypersphériques; Polynômes d'Herimite, Gauthier-Villars, Paris, 1926.

[2] B. C. Berndt, Ramanujan's Notebooks, part II, Springer, New York, NY, 1989.

[3] B. C. Berndt, Ramanujan's Notebooks, part III, Springer, New York, NY, 1991.

[4] J. M. Borwein, P. B. Borwein, Pi and the AGM: A Study in Analytic Number Theory and Computational Complexity, John Wiley \& Sons, 1987.

[5] S. Cooper, Ramanujan's theta functions, Springer, 2017.

[6] W. Duke, Some entries in Ramanujan's notebooks, Math. Proc. Cambridge Philos. Soc. 144 no. 2, 2008, 255-266.

[7] N. T. Hái, O. I. Marichev, H. M. Srivastava, A note on the convergence of certain families of multiple hypergeometric series, Journal of Mathematical Analysis and Applications 164, 1992, 104-115.

[8] R. Ito, The Beilinson conjectures for CM elliptic curves via hypergeometric functions, Ramanujan J 45, 2018, 433-449.

[9] R. Ito, The special values of $L$-functions at $s=1$ of theta products of weight 3, Research in Number Theory 5, 2019, 1-8.

[10] N. Otsubo, Certain values of Hecke L-functions and generalized hypergeometric functions, J.Number Theory 131, 2011, 648-660.

[11] M. Rogers, Boyd's conjectures for elliptic curves of conductor 11, 19, 39, 48 and 80, unpublished notes, 2010.

[12] M. Rogers, J.G. Wan, I.J. Zucker, Moments of elliptic integrals and critical L-values, Ramanujan J. 37, 2015, 113-130.

[13] M. Rogers, W. Zudilin, From L-series of elliptic curves to Mahler measures, Compositio Math. 148, 2012, 385-414.

[14] D. Samart, Three-variable Mahler measures and special values of modular and Drichlet L-series, Ramanujan J. 32, 2013, 245-268.

[15] G. Shimura, Elementary Dirichlet Series and Modular Forms, Springer, 2007.

[16] L. J. Slater, Generalized Hypergeometric Functions, Cambridge University Press, 1966.

[17] H. M. Srivastava, P. W. Karlsson, Multiple Gaussian Hypergeometric Series, Mathematics and Its Applications, Ellis Horwood, Chichester; Halsted Press (John Wiley \& Sons), New York, 1985. 
[18] J. Top, N. Yui, Congruent number problems and their variants, Algorithmic Number Theory vol. 44, 2008, 613-639.

[19] W. Zudilin, Period(d)ness of L-Values, Number Theory and Related Fields, Springer Proceedings in Mathematics and Statistics vol. 43, 2013, 381-395. 